\title{
Onychomycosis due to a rare fungus, Paecilomyces variotii - A Case Report
}

\author{
Authors
}

\section{Dr Santwana Verma ${ }^{1}$, Dr Ghanshyam Verma ${ }^{2 *}$, Dr Anumeha Gupta} Dr Amit Chauhan ${ }^{4}$, Dr Vineeta Sharma ${ }^{5}$, Dr Ankur Rana ${ }^{6}$

${ }^{1}$ Professor Microbiology, ${ }^{2}$ Associate Professor Dermatology, ${ }^{3}$ Junior Resident Microbiology

${ }^{4}$ Ex- Resident Dermatology, ${ }^{5}$ Asisitant Professor Microbiology,

${ }^{6}$ Junior Resident Microbiology, Indira Gandhi Medical College Shimla, HP, India

*Corresponding Author

Dr Ghanshyam Verma

Associate Professor, Dept of Dermatology Venereology, Indira Gandhi Medical College Shimla, HP. India

\begin{abstract}
Onychomycosis is an infection of the nails commonly caused by dermatophytes. There is a change in epidemiology of the causative agents. Non-dermatophytic fungal agents though rarely implicated are recently being recognised as nail pathogen more frequently. Paecilomyces variotii is a non-dermatophytic mold which is exceptionally reported as a cause of fungal infections. It has been rarely associated with human infections like endocarditis, endophthalmitis, pneumonia, sinusitis and cutaneous hyalohyphomycosis, invariably in poor immune responders. Onychomycosis due to Paecilomyces variotiiis rarer still as only two cases are reported earlier. The present casefocuses the relevance of recognition of an uncommon causative fungus in onychomycosis, a frequent infectionin an immunocompetent individual.
\end{abstract}

Keywords: Onychomycosis, Paecilomyces variotii, itraconazole, immunocompetent.

\section{Introduction}

Onychomycosis, fungal infection of the nail, constitutes an important public health problem because of its high prevalence and associated morbidity more so with advanced age. Prevalence in patients $60-79$ years of age is $18.2 \%$ as compared to $0.7 \%$ in subjects younger than 19 years of age ${ }^{[1]}$. The condition is characterised by irregular, brittle, discoloured and disfigured nails with complete destruction of nail plate in extensive disease. Being difficult to treat, onychomycosis may trigger more infectious lesions on other parts of the body. Dermatophyte fungi are the predominant pathogens responsible for onychomycosis. The yeasts and non dermatophytic molds including Acremonium, Alternaria, Aspergillus sp., Fusarium sp., Scytalidium and Scopulariopsis are implicated in few cases ${ }^{[2]}$. Paecilomyces variotii is an exceptional causative agent. It has been rarely associated with human infections like endocarditis, endophthalmitis, pneumonia, sinusitis and cutaneous hyalohyphomycosis, invariably in poor immune responders $^{[2,3]}$. Onychomycosis due to Paecilomyces variotii is rarer still as only two cases are reported earlier. ${ }^{[4,5]}$ This patient highlights the relevance of recognition of an uncommon causative fungus in onychomycosis which is a widespread infection. 


\section{JMSCR Vol||07||Issue||05||Page 480-483||May}

\section{Case Report}

A 65-year-old lady presented with changes in her nails of left foot. They had been discoloured brownish-yellow distally for the past 6 months. The nails had become thickened and difficult to cut. She experiences pain while walking especially in closed shoes. Recently, the patient has observed similar changes in nails of thumb and ring finger of right hand also. The patient was otherwise in good health and denied nail trauma or dystrophic abnormalities prior to the present lesions. There was no history of diseases like diabetes mellitus, psoriasis or any other conditions where nails are involved. General physical examination revealed a healthy afebrile woman with normal vitals. In physical examination, affected toes showed thickened nails with longitudinal ridges, irregular plate and a crumbling material beneath. Nail of the great toe was completely infected and weathered (Figure 1).

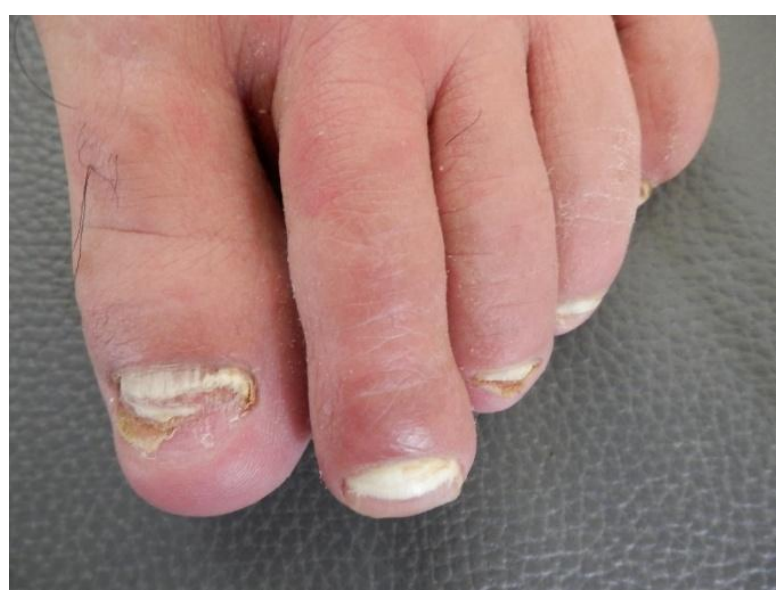

Figure 1: Great toe nail dystrophy

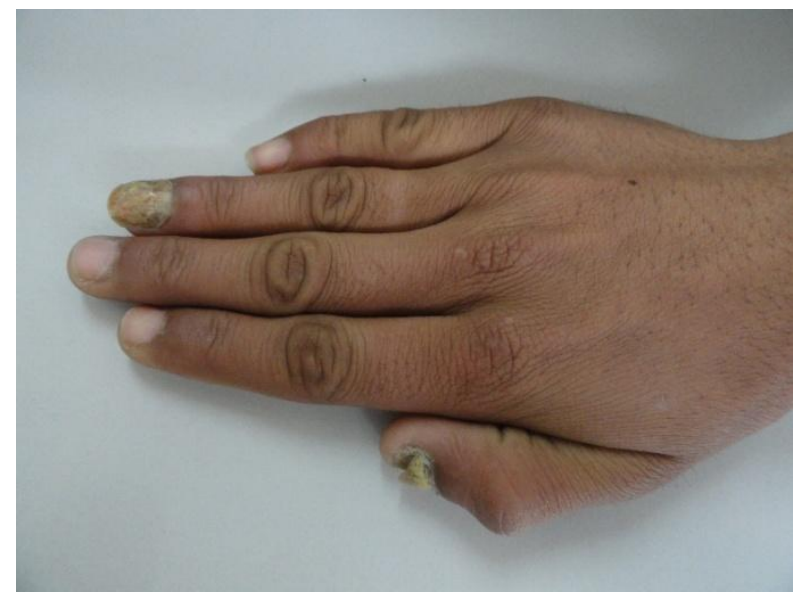

Figure 2: Finger nail involvement

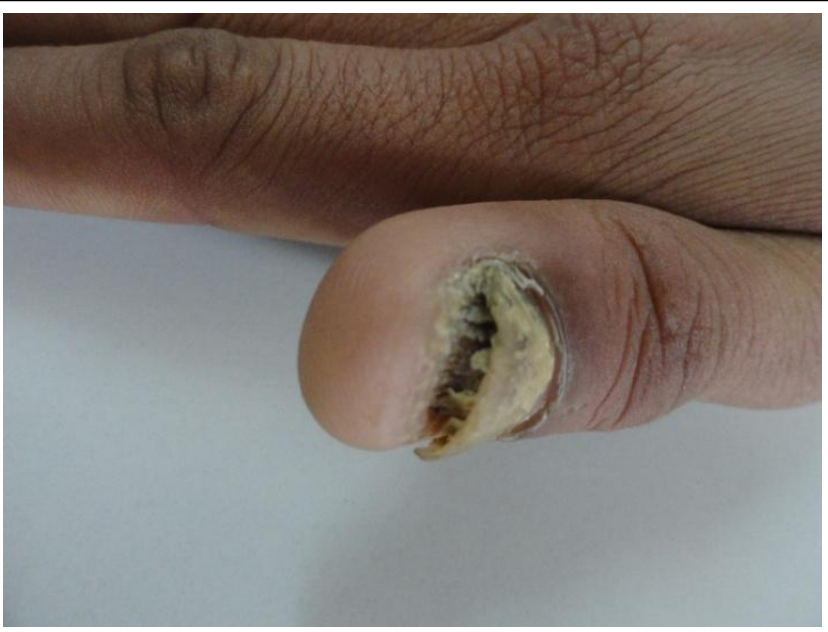

Figure 3: Dystrophic thumb nail

The thumb nail was lifted and appeared thick, brownish-yellow and nearly detached from nail-bed (Figure 2 and 3). Nails were discoloured yellow, more distally than proximally. The nearby skin was normal and no maceration was observable in the inter digital spaces. Rest of the mucocutaneous and systemic examination was non-contributory. Infected nails were clipped and subjected to direct microscopy using $40 \% \mathrm{KOH}$ which revealed hyaline septate branching hyphae. Fungal cultures from different nail specimens were performed on Sabouraud's dextrose agar with chloramphenicol at $25^{\circ} \mathrm{C}$ and $37^{\circ} \mathrm{C}$.Fast growing, powdery to floccose yellow-brown colonies were observable in 3-5 days (Figure 4).

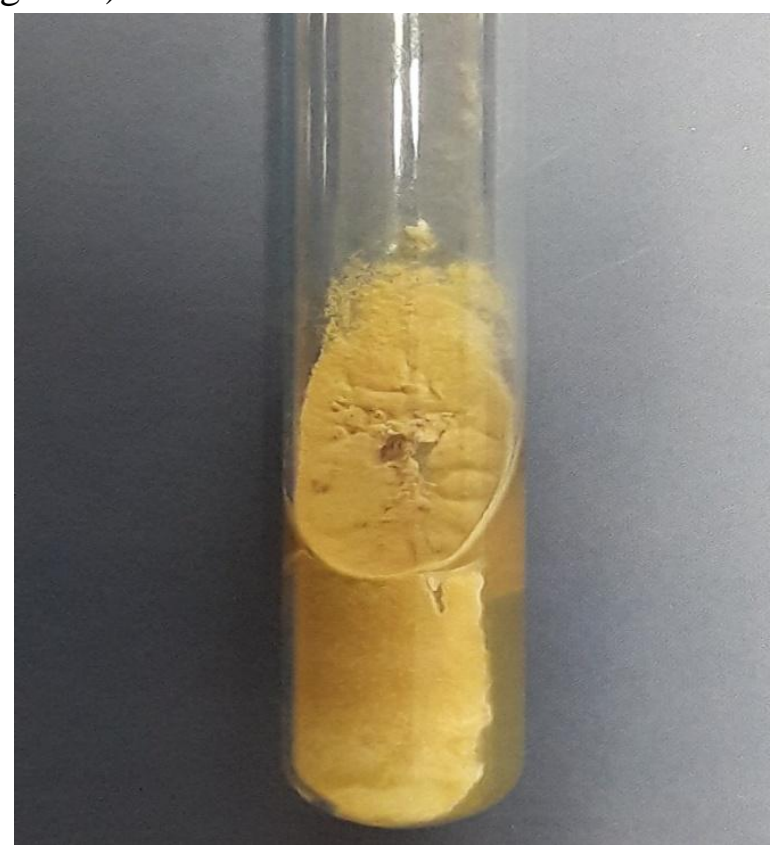

Figure 4: Powdery to floccose yellow-brown colonies on SDA media. 
Lactophenol cotton blue (LCB) wet mounts revealed characteristic hyaline, septate hyphae with conidiophores bearing dense verticillately arranged branches. Cylindrical, tapering phialides bearing smooth-walled, ellipsoidal conidia arising in long divergent chains were seen (Figure 5).

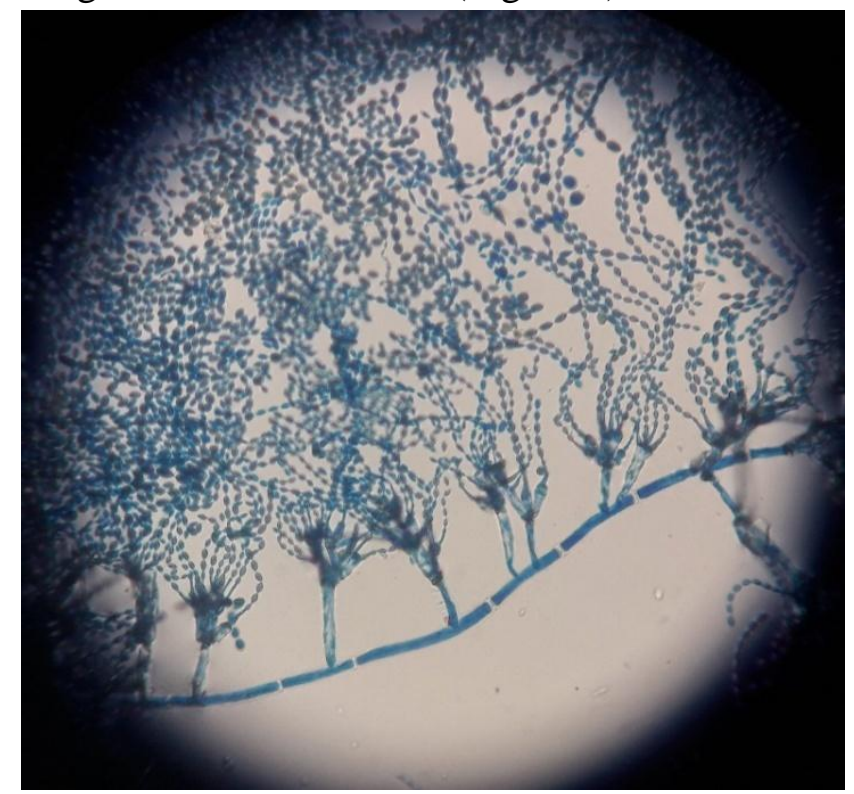

Figure 5: Lactophenol cotton blue (LCB) wet mounts revealed hyaline, septate hyphae with conidiophores bearing dense verticillately arranged branches

The isolate was dispatched to National Culture Collection of Pathogenic Fungi (NCCPF), PGIMER, Chandigarh, where identification was confirmed as P. variotii vide accession number IL3420. Patient was prescribed itraconazole100mg b.i.d. Favourable response was observed with slow clearing of infection.

\section{Discussion}

Onychomycosis is traditionally referred to nondermatophyte nail infection but lately used as a general term to illustrate all fungal infections of nails. The toenails are more often affected in a ratio of $4: 1$ to finger nails ${ }^{[6]}$. Trauma or disease-causing dystrophy of nails predisposes to infection and our patient indulging in farming as a part-time job probably acquired the infection in a similar fashion though no history of trauma was notified.

Paecilomyces species are ubiquitous soil saprophytes also obtained in cultures from indoor air, wood and pasteurized foods. It is an emerging causative agent of human mycosis ${ }^{[2,7]}$ The two species frequently implicated in human infections are P. lilacinus and P.variotii.

The significance of isolating $P$. variotii from specimens can be dubious as this is an unconventional human pathogen. Only sporadic cases are reported. The spectrum of diseases includes endocarditis, pneumonia, rhinosinusitis, endophthalmitis and ocular mycosis ${ }^{[2,3,8,9]}$. $P$. variotii has been isolated from samples of liver biopsy, vitreous aspirates, sputum, CSF, faeces and nails ${ }^{[7]}$. It is reported to colonize renal pelvis in a patient of nephrolithiasis and CSF shunts as well ${ }^{[2]}$. Onychomycosis is rarely encountered and extensive search of published reports revealed only two cases previous to the present case. ${ }^{[4,5]}$ In the present case, $P$. variotii was isolated from samples obtained from two separate infected nails and confirmation of the species was done at NCCPF, PGIMER.

Taxonomically, this hyalohyphomycotic fungus belongs to the Order Eurotiales ${ }^{[7]}$. It bears resemblance to Penicillium and Aspergillus species. The other pathogenic species, $P$. lilacinuscan be differentiated on the basis of colony colour and microscopic features. Another look-alike fungus is the Talaromyces eburneus which unlike P. variotii is not thermophilic ${ }^{[7]}$. $P$. variotii is characterised by slow growth at $25^{\circ} \mathrm{C}$, grows moderately at $28^{\circ} \mathrm{C}$ and $40^{\circ} \mathrm{C}$ and rapidly within 3 days at $37^{\circ} \mathrm{C}$. This fungal agent grows well even at temperatures as high as $50^{\circ} \mathrm{C}$ and possibly $60^{\circ} \mathrm{C}^{[10]} . P$. variotii produces fast growing, powdery to floccose yellow-brown or sand coloured colonies with a sweet odour ${ }^{[3]}$. Preliminary identification was done on colony morphology and micro-slide culture showing typical conidiophores bearing dense verticillately arranged branches with 2-5 cylindrical phialides tapering abruptly into a long thin cylindrical neck. Conidia were ellipsoidal, smooth-walled arising in long divergent chains. Subspherical chlamydospores were visualized arranged singly at places ${ }^{[3,9,11]}$

The differentiation of P.variotii from P. lilacinus becomes relevant from patient management point of view. The antifungal susceptibilities of the two are variable as $P$. lilacinus is more resistant to multiple 
antifungal drugs. $P$. variotii is uniformly sensitive to amphotericin $\mathrm{B} .^{[7,9,10]}$. Amphotericin $\mathrm{B}$ in a total dose of $1 \mathrm{gm}$ followed by itraconazole $400 \mathrm{mg}$ daily with continuation of itraconazole for five months as a prophylactic measure has been used for complete cure of severe systemic infection. ${ }^{[12]}$ Contrary to resistance in $P$. lilacinus, itraconazole and fluconazole used alone have also been found to be useful in some $P$. variotii infections. ${ }^{[12]}$ Our patient responded to itraconazole and treatment was effected by prescribing 100mg b.i.d for 6 months. Other effective azoles include ketoconazole, posaconzole, voriconazole and the novel triazole UR-9825 $5^{[12]}$. In vitro testing has demonstrated susceptibility to terbinafine ${ }^{[10,13]}$. Interestingly, echinocandins like caspofungin and especially micafungin and anadulafungin are highly active agents $^{[7]}$.

The consciousness to the pathogenic role of $P$. variotii is essential for the clinician and microbiologist to spot this fungal agent as pertinent and successfully treat both mild and serious infections when encountered.

\section{References}

1. Ghannoum $\mathrm{M}$ and Isham N. Fungal nail infections (Onychomycosis): A neverending story.PLoS Pathogens. 2014;10(6): e1004105.

2. Kwon-Chung KJ, Bennett JE. Infections due to miscellaneous molds. In: Kwon-Chung KJ, Bennett JE, editors. Medical Mycology. Philadelphia, USA: Lea and Febiger 1992.p.733-67.

3. DeHoog GS, Guarro J, Tan CS, Wintermans RGF, Gene J. Rare opportunistic fungi. In: DeHoog GS, Guarro J, editors. Atlas of Clinical Fungi. The Netherlands: Centraalbureau voor Schimmel culture, Baarn and Delft1995.p.580-81.

4. Arenas R, Arce M, Munoz H, Esmenjaud JR. Onychomycosis due to Paecilomyces variotii. A case report and review. J Med Mycol.1998;8(1):32-33.
5. Fletcher CL, Hay RJ, Midgley G, Moore M. Onychomycosis caused by infection with Paecilomyces variotii. Br J Dermatol. 1998; 139:1133-1135.

6. Jagdish Chander. Dermatophytosis. In: Jagdish Chander, editor. Textbook of Medical Mycology. New Delhi, India: Jaypee Brothers Medical Publishers(P)Ltd. 2018.p.162-200.

7. Houbraken J, Verweij PE, Rijs AJMM, Borman AM, Samson RA. Identification of Paecilomyces variotii in clinical samples and steeings. J Clin Microbiol. 2010;48 (8):2754-2761.

8. Swami T, Pannu S, Kumar M, Gupta G. Chronic invasive fungal rhinosinusitis by Paecilomyces variotii: A rare casereport. Indian JMed Microbiol2016; 34:103-106.

9. Lam DSC, Koehler AP, Fan DSP, Cheuk W, Leung AST, Ng JSK. Endogenous fungal endophthalmitis caused by Paecilomyces variotii.Eye.1999; 13:113-116.

10. Salle V, Lecuyer E, Chouaki T, Lescure FX, Smail A, Vaidie A, et al. Paecilomyces variotii fungemia in a patient with multiple myeloma: case report and literature review. J I nfect. 2005; 51: e93-e95.

11. Abbas SQ, Maan A, Iqbal J, Niaz M, Waqas $\mathrm{M}$, Ahmed $\mathrm{H}$, et al. A report of Paecilomyces variotii on human from Pakistan. Pak J Bot. 2009;41(1):467-472.

12. Lee J, Yew WW, Chiu CSW, Wong PC, Wong CF, Wang EP. Delayed sternotomy wound infection due to Paecilomyces variotii in a lung transplant recipient. J Heart Lung Transplant 2002; 21:1131-1134.

13. Steiner B, Aquino VR, Paz AA, Silla LMR, Zavascki A, Goldani LZ. Paecilomyces variotii as an emergent pathogenic agent of pneumonia. Case Rep Infect Dis2013; 2013:273848. 